\title{
Persönliche Schutzausrüstung an- und ausziehen - Schritt für Schritt
}

Marcus Reska, Michaela Berger

\author{
Es heißt: „Eine schöne Krawatte zieht die Tagessuppe magisch an“. Im Kranken- \\ hausalltag geht es um mehr als Suppe: Das Risikospektrum reicht von luftübertrag- \\ baren Erkrankungen wie Tuberkulose bis zum schwallartigen Erbrechen bei Patienten \\ mit Norovirusinfektion. Zum Schutz vor Infektionen, Chemikalien oder Erreger- \\ verbreitung ist die persönliche Schutzausrüstung unerlässlich und effizient - \\ entscheidend dabei ist das Wie des An- und Ausziehens.
}

\section{Persönliche Schutzausrüstung kurz und knapp}

Charakteristisch für die persönliche Schutzausrüstung (PSA) ist ihre spezielle Schutzfunktion vor einem oder mehreren Risiken. Von vielen wird die PSA wertgeschätzt als Schutz vor Infektionsrisiken. Für andere stellt sie eine lästige Notwendigkeit und Behinderung des Arbeitsflusses - bei ohnehin schon hohem Zeitdruck und Personalmangel - dar. Gerade Zeitmangel in Kombination mit vermeintlich einfach erscheinenden Tätigkeiten - wie das An- und Ablegen der PSA - birgt Risiken für Fehler bei der Durchführung.

Detaillierte Hinweise zur konkreten Benutzung persönlicher Schutzausrüstung liefern unter anderem

- die Technische Regel für Biologische Arbeitsstoffe 250 (TRBA 250),

- die Empfehlung der Kommission für Krankenhaushygiene und Infektionsprävention (KRINKO) beim Robert Koch-Institut „Infektionsprävention im Rahmen der Pflege und Behandlung von Patienten mit übertragbaren Krankheiten“,

- die Vorschriften und Regelwerke der Deutschen Gesetzlichen Unfallversicherung (DGUV) sowie das Medienportal der zuständigen Berufsgenossenschaft/ Unfallkasse.

\section{Atemschutzmaske}

Die Atemschutzmaske hat generell die Aufgabe, vor in der Atemluft befindlichen Schadstoffen (z. B. auch Mikroorganismen) zu schützen. Wie gut sie das macht, hängt bei den partikelfiltrierenden Halbmasken (engl. Filtering Face Pieces, FFP) von der Gesamtleckage ab, sprich der Summe von Undichtigkeiten.
Verantwortlich dafür sind neben möglichen Undichtigkeiten am Ausatemventil hauptsächlich 2 Faktoren:

- Die Verpassungsleckage: Sie vergrößert sich, je größer die Undichtigkeiten an den Stellen sind, an denen die Maske am Gesicht aufliegt (Dichtlinie).

- Der Filterdurchlass: Je geringer er ist, desto größer ist die Effizienz der Abscheidung von Partikeln und Vermeidung der kompletten Durchdringung. Dafür verantwortlich sind z.B. Schichtdicken und elektrische Fasereigenschaften.

Partikelfiltrierende Halbmasken werden auf der Basis ihrer Gesamtleckage in 3 Klassen unterteilt:

- FFP 1 (max. 22\%)

- FFP 2 (max. $8 \%$ )

- FFP 3 (max. 2\%)

Neben den aktuellen Empfehlungen und Vorgaben zur Nutzung und Auswahl von Atemschutzmasken sollten immer das tatsächlich vorhandene Infektionsrisiko, die Praktikabilität und Mitarbeiter-Compliance mit berücksichtigt werden. Der fachliche Austausch zwischen dem Arbeitsschutz und der Hygiene ist sinnvoll.

\footnotetext{
Merke

Dient die Maske dem Schutz vor luftübertragbaren Erregern oder Aerosolen, wird mindestens eine FFP-2-Maske verwendet. Insbesondere hier sollte nach Anlegen dieser Maske eine Dichtsitzprüfung durchgeführt werden (s.u.).
}

Für Bartträger ist zu beachten: Vermehrter Haarwuchs im Bereich der Dichtlinie kann zu einer Leistungsminderung der Atemschutzmaske führen und damit das Gesundheitsrisiko erhöhen - insbesondere bei Indikationen für einen höherwertigen Atemschutz (FFP 2, FFP 3). 


\section{Schutzhandschuhe}

Schutzhandschuhe stellen einen Schutz vor Kontakt mit Körperflüssigkeiten, Ausscheidungen oder flüssigen Chemikalien dar. Diese können über verschiedene Wege über die Haut der Hände direkt oder sekundär über die Hände als Vehikel in den Kreislauf gelangen - z. B. über Stichverletzung, bestehende Wunden oder Risse trockener Haut, durch während des Handschuhausziehens kontaminierte Hände.

Schutzhandschuhe kommen mit unterschiedlichen Materialeigenschaften und Anforderungen an die jeweilige Anwendung zum Einsatz. So sollten z. B. immer der abzuwehrende Stoff und die möglichen mechanischen Beanspruchungen mit in die Auswahl einfließen.

\section{Merke}

Vor der Entnahme von Handschuhen aus den gewöhnlichen Pappboxen sind zur Vermeidung einer Kontamination von Box und Handschuhen die Hände zu desinfizieren.

\section{Schutzkittel}

Der Schutzkittel dient generell dem Schutz des Mitarbeiters vor Kontamination seiner Bereichs-/Arbeitskleidung mit Infektionserregern. Der Schutzkittel wird also zusätzlich und situationsbezogen angezogen. Prinzipiell ist er langärmelig und hinten geschlossen. Die Auswahl der Art des Kittels richtet sich nach der Tätigkeit, dem Kontaminationsrisiko und dem möglichen Durchfeuchtungsgrad.

\section{Schritt für Schritt}

Häufig besteht die Indikation für das gleichzeitige Tragen von Schutzkittel, Atemschutz und Handschuhen. In welcher Reihenfolge das An- und Ablegen durchzuführen und was dabei zu beachten ist, wird nachfolgend beispielhaft beschrieben.

\section{Schritt 1 Anlegen des Schutzkittels}

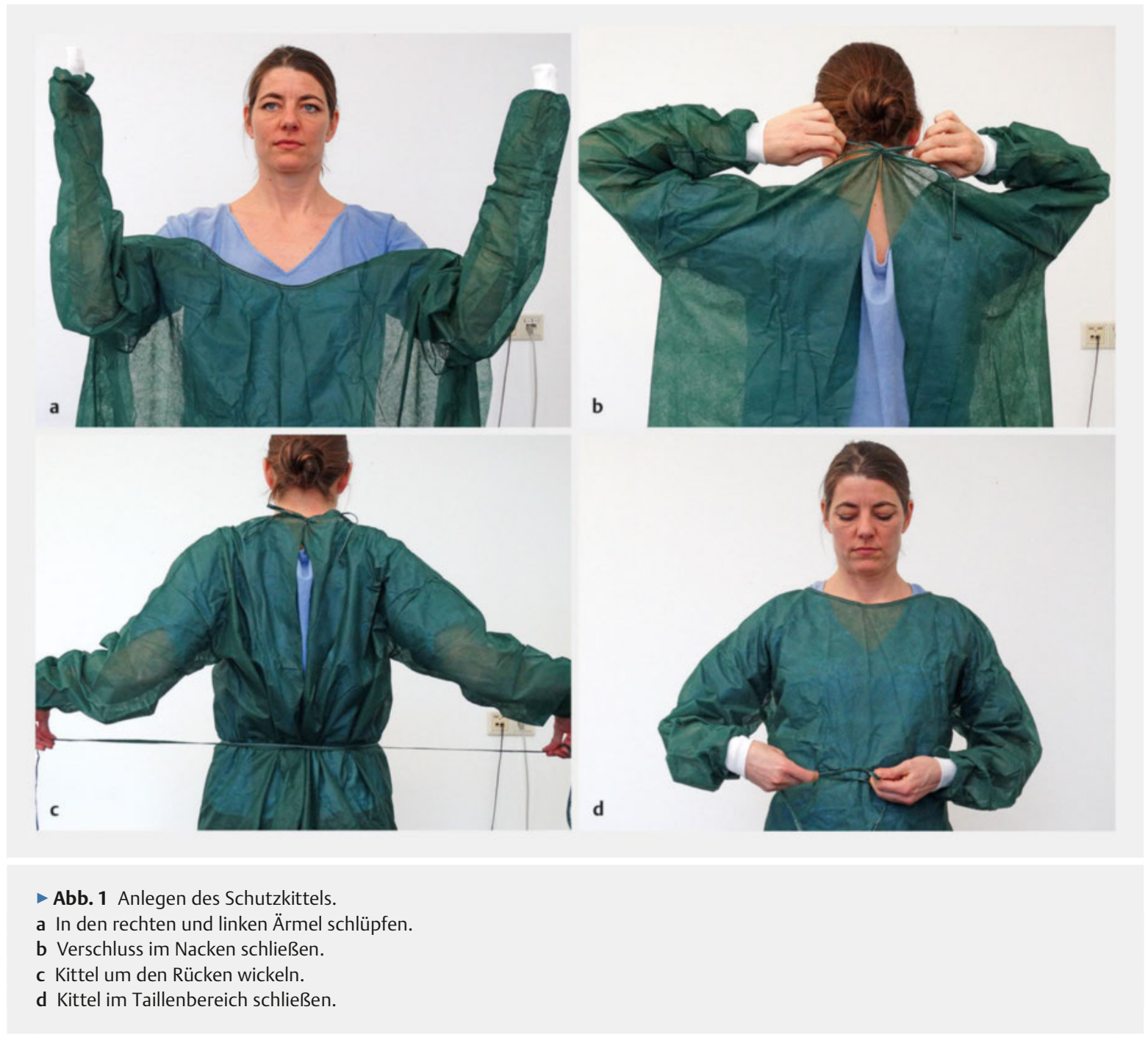


- Entnehmen Sie mit den (sauberen, desinfizierten) Händen den Schutzkittel.

- Schlüpfen Sie jeweils in den rechten und linken Ärmel und ziehen den Kittel ganz bis über die Schultern hinweg.

- Der Kittel bedeckt nun den ganzen Körper vom Hals bis zu den Knien und die Arme bis zum Handgelenk.

- Wickeln Sie den Kittel um den Rücken und schließen Sie ihn mittels der Verschlüsse im Nacken- und Taillenbereich.

\section{Schritt 2 Anlegen der Atemschutzmaske}

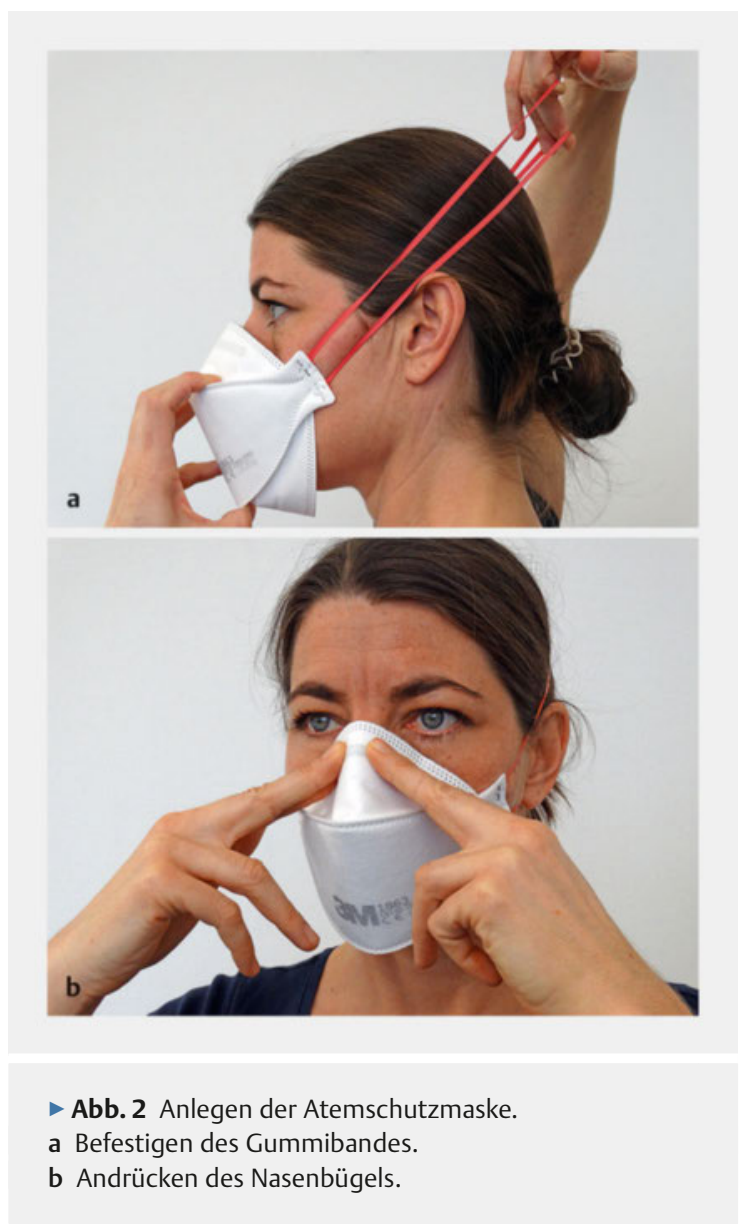

- Befestigen Sie die Atemschutzmaske mit den Verschlussbändern bzw. dem Gummiband an der hinteren Kopfmitte und im Nackenbereich.

- Drücken Sie den Nasenbügel so an, dass möglichst keine Leckagen entstehen.

- Positionieren Sie die Maske angenehm im Gesicht und unterhalb des Kinns.

- Dichtsitzprüfung (Fit-Check) - insbesondere für die Verwendung der Atemschutzmaske mit einer Indikation der Filterleistung ab FFP 2: Legen Sie beide Handflächen abdeckend auf die Halbmaske. Atmen Sie anschließend tief ein und aus. Wenn Sie spüren, wie Ihre ausgeatmete Luft schnell an den Maskenrändern entweicht (besonders im Bereich Kinn und Nasenrücken), passen Sie die Maske erneut an.

- Nehmen Sie die Maske nicht zwischendurch ab und lassen Sie sie nicht „lätzchenartig“ hängen, um sie danach wieder aufzusetzen! Stattdessen Maske wie unten ausgeführt ausziehen und eine neue anlegen!

- Lagern Sie die Masken trocken und staubgeschützt.

\section{Schritt 3 Anlegen der Schutzhandschuhe}

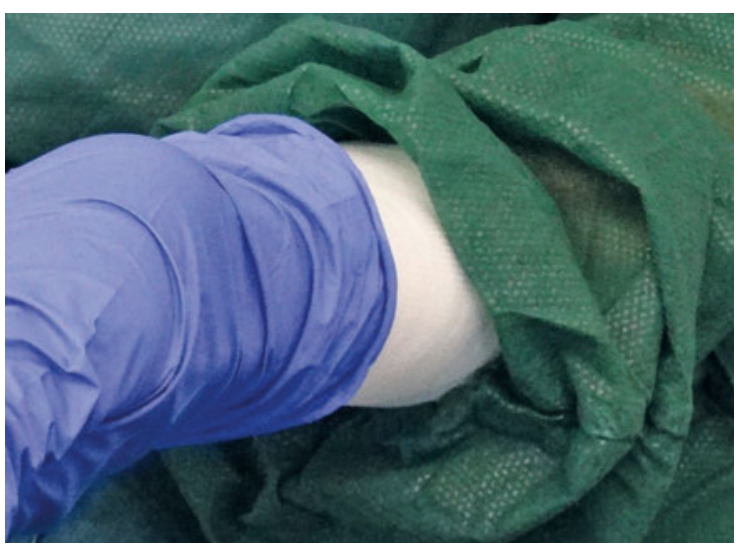

- Abb. 3 Anlegen der Schutzhandschuhe. Der Handschuh wird über das Handgelenk und den Schutzkittelbund gezogen.

- Entnehmen Sie zunächst nur einen Schutzhandschuh aus der Handschuhpackung.

- Stülpen Sie diesen Schutzhandschuh zunächst auf die dominante Hand. Achten Sie darauf, dabei möglichst wenig der Handschuhaußenfläche zu berühren und Kontakt zum Gesicht und anderen Oberflächen zu vermeiden.

- Ziehen Sie den Handschuh über das Handgelenk und den Schutzkittelbund. 
- Entnehmen Sie nun mit der behandschuhten Hand den zweiten Handschuh.

- Halten Sie den Handschuh an der Stulpe fest, während der Handschuh über die andere Hand und den Schutzkittelbund gestülpt wird.

- Nun können beide Handschuhe im Sitz angepasst werden.

\section{Cave}

- Entnehmen Sie Handschuhe immer mit sauberen und desinfizierten Händen aus der Spenderbox.

- Stecken Sie versehentlich entnommene Handschuhe nicht wieder zurück in die Spenderbox (potenziell kontaminiert).

\section{Schritt 4 Ablegen der \\ Schutzhandschuhe}

- Heben Sie einen Handschuh mit der anderen Hand an der Stulpenaußenseite etwas an, ohne dabei die Haut zu berühren (Außenseiten potenziell kontaminiert!).

- Ziehen Sie nun den Handschuh vom Handgelenk runter zu den Fingern. Dabei wird die Handschuhinnenseite nach außen gekehrt und der gesamte Handschuh von der Hand abgerollt.

- Halten Sie den abgerollten Handschuh in der noch behandschuhten Hand.

- Die nicht behandschuhte Hand fasst jetzt unter die Stulpe der behandschuhten Hand. Der Handschuh wird ebenfalls so abgezogen, dass seine Innenseite nach außen gekehrt und über den noch in der Hand gehaltenen, bereits abgerollten Handschuh gezogen wird.

- Entsorgen Sie beide Handschuhe.

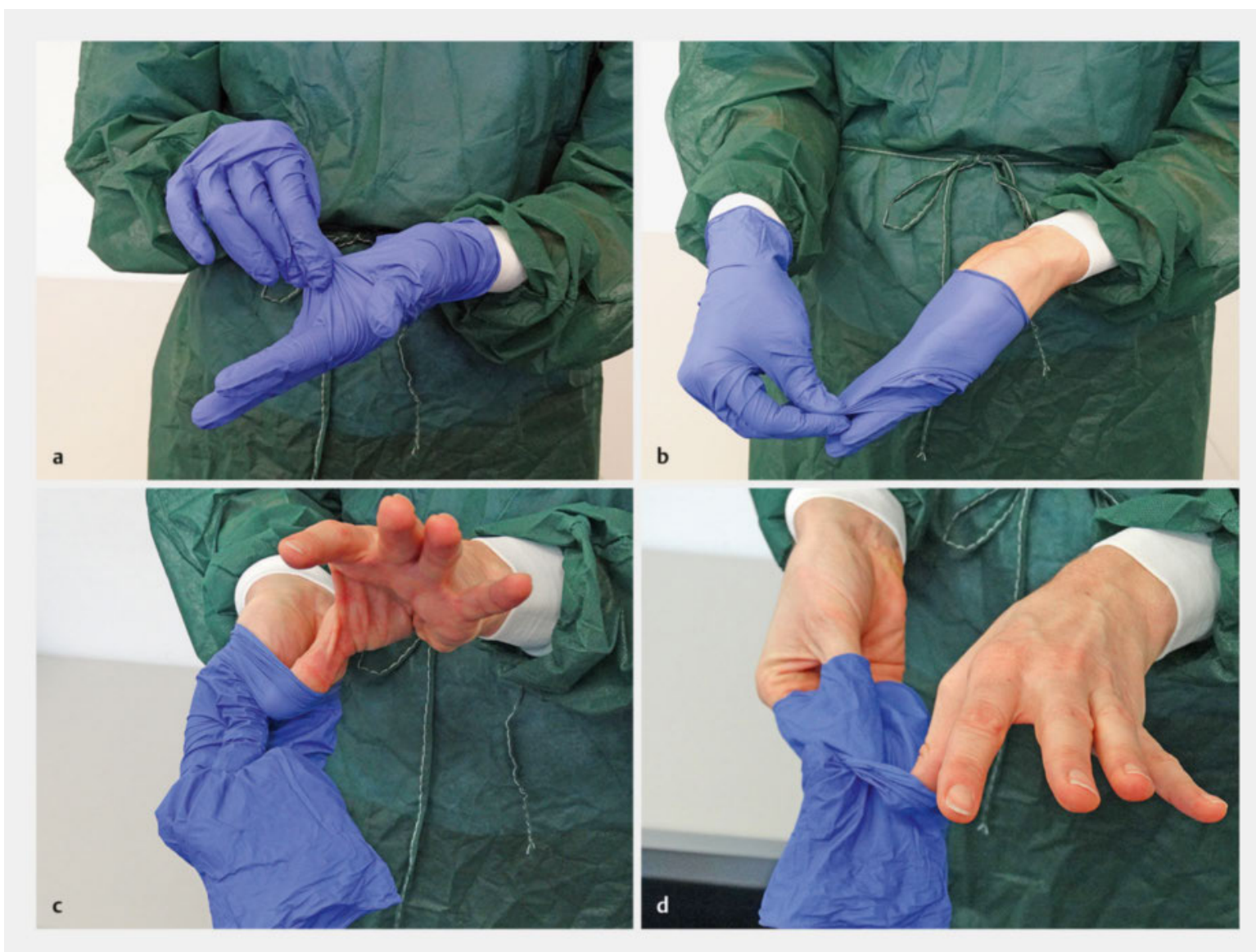

- Abb. 4 Ablegen der Schutzhandschuhe.

a Anheben eines Handschuhs mit der anderen Hand.

b Abrollen des Handschuhs von der Hand mit der Innenseite nach außen.

c Mit der nicht mehr behandschuhten Hand unter die Stulpe des zweiten Handschuhs fassen.

d Abziehen des zweiten Handschuhs mit der Innenseite nach außen über den ersten Handschuh. 


\section{Cave}

Auch während des Tragens und des Ausziehens von Schutzhandschuhen kann eine Kontamination der Hände erfolgen. Eine Händedesinfektion ist deshalb nach Ablegen der Schutzhandschuhe immer obligat.

\section{Schritt 5 Ablegen des Schutzkittels}

- Lösen Sie zunächst die Nacken- und Taillenverschlüsse (Außenseiten potenziell kontaminiert!).
- Lassen Sie durch eine schälende Bewegung die Oberhälfte des Kittels herab.

- Greifen Sie mit der linken Hand die linke Innenseite und mit der rechten Hand die rechte Innenseite im Schulterbereich.

- Ziehen Sie den Kittel hinunter, dessen Innenseite nun nach außen gekehrt ist.

- Halten Sie den Kittel vom Körper fern, während sie ihn zu einem Bündel geformt entsorgen.

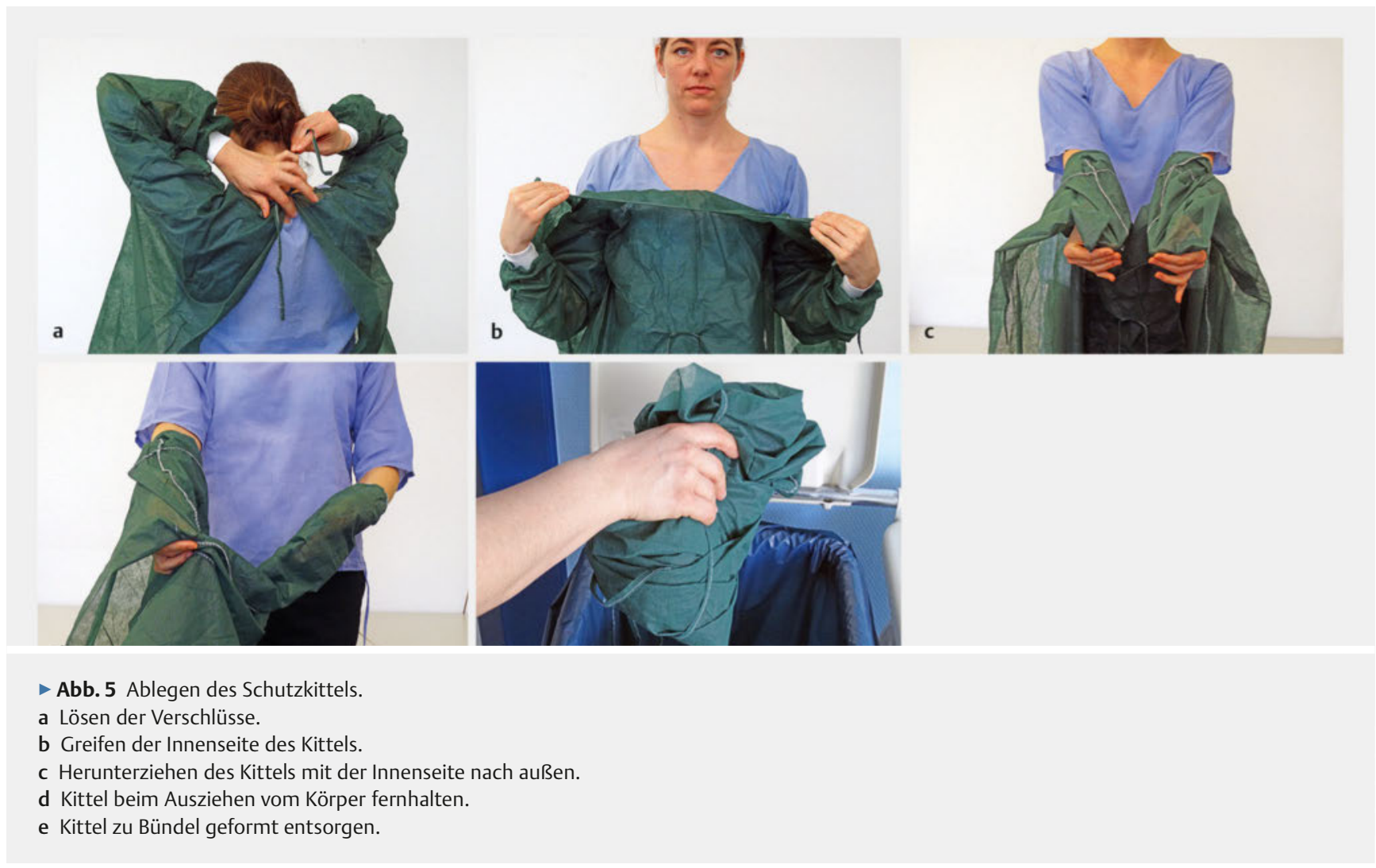




\section{Schritt 6 Ablegen der Atemschutzmaske}

- Berühren Sie möglichst nicht die potenziell kontaminierte Maske.

- Greifen Sie mit sauberen und desinfizierten Händen zunächst das untere Gummiband in Nackenhöhe.

- Greifen Sie anschließend zusätzlich das Gummiband, das sich auf Höhe der Kopfmitte befindet.
- Ziehen Sie jetzt beide Bänder über den Kopf hinweg nach vorne.

- An beiden Bändern haltend, können Sie nun die Maske vom Gesichtsfeld lösen.

- Entsorgen Sie die Maske.

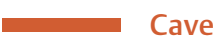

Je nach Maskentyp ist ein Berühren der Maskenvorderseite unvermeidlich.
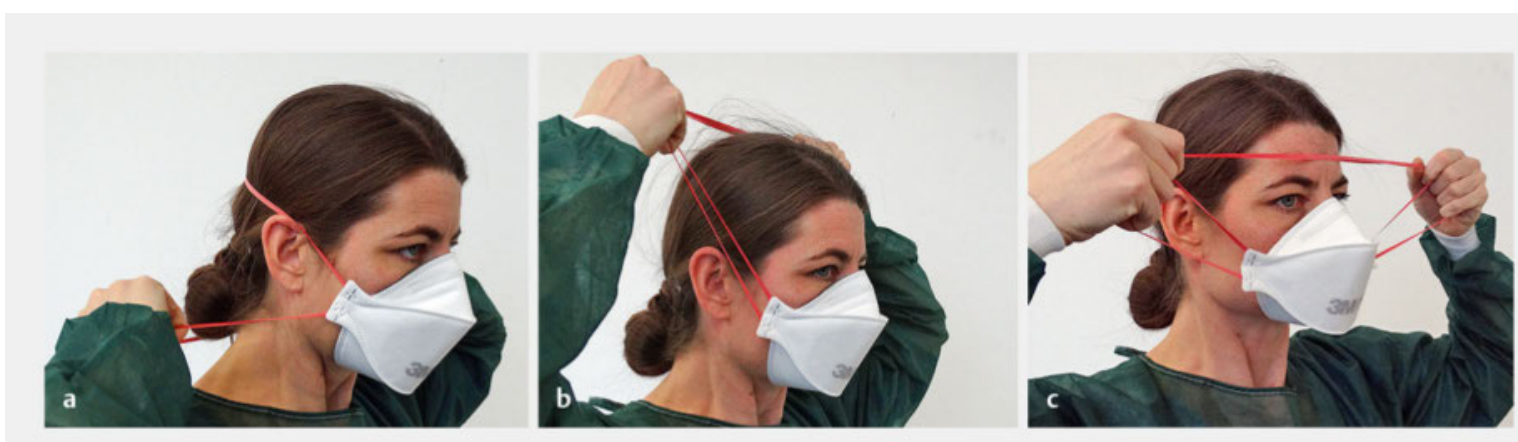

- Abb. 6 Ablegen der Atemschutzmaske.

a Greifen des unteren Gummibandes in Nackenhöhe.

b Beide Bänder über den Kopf hinweg nach vorne ziehen.

c Die Maske - an beiden Bändern festhaltend - vom Gesicht lösen.

Interessenkonflikt

Die Autoren geben an, dass kein Interessenkonflikt besteht.

Über die Autoren

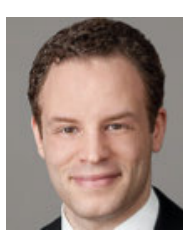

\section{Marcus Reska}

Dr. rer. nat. Dipl.-Biol., Jahrgang 1977. Studium der Biologie an der RWTH Aachen. 2006 Mitarbeiter am Institut für Pathologie Uniklinik Aachen. 2007-2013 wissenschaftlicher Mitarbeiter am Institut für Arbeitsmedizin und Sozialmedizin der Uniklinik Aachen. 2010

Zusatzausbildung zur Fachkraft für Arbeitssicherheit. 2011 Promotion. Seit 2013 Mitarbeiter am Deutschen Beratungszentrum für Hygiene (BZH GmbH).

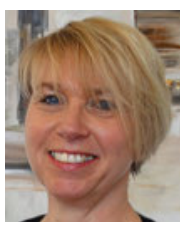

\section{Michaela Berger}

1985-2008 Gesundheits- und Krankenpflegerin. Seit 2009 Fachkraft für Hygiene und Infektionsprävention beim Deutschen Beratungszentrum für Hygiene in Freiburg.
Korrespondenzadresse

\section{Dr. rer. nat. Dipl. Biol. Marcus Reska}

Deutsches Beratungszentrum für Hygiene

Schnewlinstraße 10

79098 Freiburg

Reska@bzh-freiburg.de

Bibliografie

DOI https://doi.org/10.1055/s-0043-104957

Krankenhaushygiene up2date 2017; 12: 117-122

(c) Georg Thieme Verlag KG Stuttgart · New York ISSN 1862-5797 